\title{
A CERTAIN KIND OF FORMAL THEORIES
}

\author{
KATUZI ONO
}

\section{Introduction}

A common feature of formal theories is that each theory has its own system of axioms described in terms of some symbols for its primitive notions together with logical symbols. Each of these theories is developed by deduction from its axiom system in a certain logical system which is usually the classical logic of the first order.

There are many formal theories of mathematics, e.g. the natural number theory, the Euclidean geometry, the set theory, etc. Naturally, a single theory can be formulated in various ways just as we can describe a theory in various languages. There are, however, many mathematical theories which seem essentially different to each other; for instance, the natural number theory seems quite different from the Euclidean geometry. On the other hand, these formal theories are linked together closely by some basic theory, usually the set theory. The linkage is exhibited in reducing consistency of each theory meta-theoretically to consistency of the basic theory. We can really confirm meta-theoretically consistency of the natural number theory as well as the Euclidean geometry by assuming that the set theory is consistent. In the course of such meta-theoretical reasoning, the dominion of the basic theory is enlarged step by step. As a matter of course, we have been seeking for a consistent basic system as simple and as dominant as possible, and we have composed our trial systems $\mathbf{O Z}$ and OF along this line. (See Ono [9] and [10].) Anyway, our chief concern has been the axiom systems of formal theories, not their logic. The logic of the basic theory has been supposed to remain unchanged.

However, is it really our natural way of free thinking to develop special theories in a certain basic theory without bringing up logic itself? In the primitive stage, our logic would concern with real objects only; while in the developed stage, our logic must concern with abstract objects in the world of possibility. Are our logics quite the same for real objects and for abstract objects? Even

Received February 5, 1964. 
the logical notion existence does not remain the same in examples for real objects such as "there are books on the table" and in examples for abstract objects such as "there are complex numbers". What we have in our mind in the latter example would be only that our imagination of complex numbers leads to no contradictory circumstances at all, while in the former example we positively assert that books really exist on the table. The logic for abstract objects must be different from the primitive logic for real objects in some respect.

As well known, we can formulate the logical system for abstracts objects. as the classical logic of the first order quite independently of primitive logic for real objects. We believe, however, we had better formulate the primitive logic for real objects at first and the logic for abstract objects thereafter as a natural development of the former.

It is our opinion that the primitive logic can be developed to the logic for a developed formal theory only in connection with the primitive stage of the formal theory. In our real free thinking, we believe, our logic in the beginning is something like the positive logic without the notion of negation. Our real concern at that stage would be whether our imaginations and speculations lead to some undesirable circumstances, or metaphorically speaking, to some taboos.

These taboos can be regarded as taboos of a certain theory to be developed. However, logical denial of a proposition is surely motivated if the proposition leads to some taboos. It is a standard course of our logical denial activity that we deny a proposition because it leads to a taboo. From this point of view, taboos look like bridges between logic and special theories. Having taboos at hand, we need not start from a very complete logical system such as the predicate logic of the first order. Possibly, we can start from a simple logical system even without negation. The positive logic introduced in $\S 3$ is a typical system of such kind. It has only two primitive notions, implication and universal quantification. The chief concern of the present paper is to study the general feature of developments of formal theories starting from the primitive positive logic and the taboo systems of the respective formal theories.

In dealing with logical problems, we have to consider matters standing on various levels as occasions require. However, even when we discuss logical problems, we seldom state clearly on which level we are standing. We mạy 
usually find out the level from contexts, but we may as well establish a convention in employing words to express clearly on which level we are standing. We discuss the matter in $\S 1$ as preliminaries.

Any logical system can be described in various manners. We have introduced a practical way of describing formal deductions (Ono [11]), and in the present paper, we would like to describe logical systems mostly along our practical way. However, any logical system, even such a popular system as the classical logic of the first order, has not been exactly exhibited in our practical way. In $\S 3$, we describe a system of positive logic along our practical way as well as along Gentzen's way (Gentzen [2]), and prove equivalence of the both descriptions. To bring out the mutual relation of the two descriptions as clearly as possible, our system is slightly modified so as to be able to describe proofs of sequents. A thorough exposition of the modified system is given in $\S 2$.

The proof of equivalence of descriptions of the positive logic in our practical way and in the Gentzen manner deserves special attention, because the proof leads to the conclusion that a system introduced in $\S 4$, a development PLK of the positive logic, is essentially nothing but the ordinary classical predicate logic of the first order. In $\S 4$, a few other systems are introduced, which are in close connection with the minimal logic (See Johansson [6].) and the intuitionistic logic (Compare Glivenko [3] and also Kuroda [7]).

In the last $\$ 5$, we introduce a few examples of taboo systems. Most of the formal theories defined by axiom systems would be defined also by taboo systems. We are dreaming that a certain basic theory would be defined by a really simple taboo system. To formulate taboos in simple forms in the primitive positive logic is far more difficult to formulate axioms in simple forms in the ordinary predicate logic, because primitive pcsitive logic allows smaller vocabulary of logical terms. We believe, however, we can really formulate an object theory equivalent to $\mathrm{OF}$ by a taboo system which can be described as simply as the axiom schemes of $\mathbf{O Z}$ or $\mathbf{O F}$.

\section{Preliminaries}

When we talk on logical problems, we have to do with the same words such as "assumption", "implication", "set", etc. in different meanings according 
as the level we are standing on as occasions require.

Any formal theory has some logical notions such as implication or universal quantification on the formal-theoretical level. The same words, however, can also be employed for expressing corresponding notions of higher levels. To avoid confusion, we might as well establish a convention to underline a word by a single straight line when we wish to express clearly that the word is employed standing on the formal-theoretical level.

Logic has mostly to do with propositions of formal theories standing on a higher level which could be called meta-formal-theoretical level or logical level. Typical talks standing on the logical level are such talks as "The set of assumptions lears to the conclusion". This kind of talks is nicely expressed in the sequent form $\left\{\mathfrak{A}_{1}, \ldots, \mathfrak{A}_{n}\right\} \mid=\mathfrak{B}$ or $\Gamma \mid=\mathfrak{B}^{1)}$ (We denote by a capital Greek letter a finite set of propositions including the vacant case.). To express clearly that a word denotes a notion of the logical level, we underline the word by double straight lines.

Chief concern of logic is provability, provability of propositions as well as provability of sequents. In this work, we call our attention rather to the provability of sequents. However, we call a proposition $\mathfrak{B}$ provable, when the sequent $\emptyset \mid=B$ is provable. To deal with provability of a sequent, we have to stand on a still higher level, which we call meta-logical level. To express clearly that a word denotes a notion of the meta-logical level, we underline the word by a single wavy line.

One might occasionally need to use words of so-to-say meta-meta-logical level. Such a circumstance could be clearly expressed by underlining the words by double wavy lines.

Now, provability of a sequent depends on logical systems. In any system, however, tautology and modus ponens could be assumed. These can be naturally expressed as

(Tautology) $\quad\left\{\mathfrak{A}_{1}, \ldots, \mathfrak{A}_{n}, \mathfrak{B}\right\} \mid=\mathfrak{B}$ or $\Gamma \cup\{\mathfrak{B}\} \mid=\mathfrak{B}$.

$(\mathrm{Cut})^{2)}$

$$
\frac{\left\{\mathfrak{A}_{1}, \ldots, \mathfrak{P}_{m}\right\}\left|=\mathfrak{S},\left\{\mathfrak{B}_{1}, \ldots, \mathfrak{B}, \mathfrak{S}\right\}\right|=\mathfrak{D}}{\left\{\mathfrak{H}_{1}, \ldots, \mathfrak{H}_{m}, \mathbb{H}_{1}, \ldots, \mathfrak{B}_{n}\right\} \mid=\mathfrak{D}}
$$

1) Compare Gentzen [2]. Gentzen denotes this logical relation in the form $\mathfrak{A}_{1}, \ldots, \mathfrak{A}_{n} \rightarrow \mathfrak{B}$ or $\Gamma \rightarrow \mathfrak{B} . \mathfrak{A}_{1}, \ldots, \mathfrak{A}_{n}$ or $\Gamma$ in Gentzen's formulation are not regarded as sets, but as sequences.

2) Gentzen's Schnitt. See Gentzen [2]. 
or

$$
\frac{\Theta|=\subseteq \quad A \cup\{\mathfrak{S}\}|=\mathfrak{D}}{\Theta \cup \Lambda \mid=\mathfrak{D}} \text {. }
$$

Thus far, we have nothing to do with special notions of the formal theoretical level. Concerning these notions, we believe that even the simplest logical system should have implication or something like a prototype of it. We shall denote this by $\supset$.

Naturally, implication is closely connected with the implication $\mid=$. As it is a natural way of talking to say " $\mathfrak{A}$ implies $\mathfrak{B}$ under the set of assumptions $\left\{\mho_{1}, \ldots, \mathfrak{\mho}_{n}\right\}$ " instead of "The set of assumptions $\left\{\mho_{1}, \ldots, \mho_{n}, \mathfrak{H}\right\}$ implies $\mathfrak{B}$ ", so the daily usage of the word "implication" harmonizes well with the following stipulation: $I \mid=\mathfrak{Q} \supset \supset \mathfrak{B}$ is called provable if and only if $\Gamma \cup\{\mathfrak{A}\} \mid=\mathfrak{B}$ is provable.

Usually, our logical system is expected to be a predicate logic. As we have no device to introduce quantification exclusively by proposition-logical notions, we wish to have at least one kind of quantifications to begin with. However, we can hardly define provability of a sequent with respect to quantifications so as to harmonize very well with the common-sense usages of the words such as "any", "all", "there exist", etc.

Concerning abstract objects, the meaning of existential quantification looks far more oblique than the meaning of universal quantification. In fact, we always wondering whether mathematicians could come to a unanimous agreement concerning the real meaning of the simplest statements in mathematics such as "there are negative numbers" or "there is at least one infinite set". So, let us take up another simpler example, the universal quantification.

Universal quantification is expressed here in the form $(x) \mathfrak{A}(x)$. To be simple, let us put aside any discussion on the range of a quantification, and let us take up the exemplar proposition "The natural number $x$ is even or odd" as $\mathfrak{A}(x)$. Then, the common-sense interpretation of $(x) \mathfrak{A}(x)$ must be " 0 is even or odd", " 1 is even or odd", "2 is even or odd", . . , i.e. $\mathfrak{A}(0), \mathfrak{A}(1), \mathfrak{A}(2), \ldots$

Now, how do we prove $(x) \mathfrak{A}(x)$ ? Surely, we do not prove $(x) \mathfrak{A}(x)$ by really proving $\mathfrak{H}(0), \mathfrak{A}(1), \mathfrak{A}(2), \ldots$ all. A typical device in mathematics for enabling such kind of proofs is employment of free variables denoting an arbitrary object. (Compare Suetuna's beliebig allgemein and beliebig bestimmt. See Suetuma [12].). We know that exemplar cases $\mathfrak{A}(0), \mathfrak{A}(1), \mathfrak{A}(2), \ldots$ are 
all provable, if we know that $\mathfrak{f}(x)$ is provable for any variable (not for any object). Although it is impossible to prove literally all propositions $\mathfrak{A}(0), \mathfrak{A}(1)$, $\mathfrak{Y}(2), \ldots$, but it is still possible to know for a certain kind of propositions $\mathfrak{H}(x)$ that $\mathfrak{H}(\boldsymbol{x})$ is provable for any variable $x$.

Of course, it must be impossible to prove $\mathfrak{A}(x)$ literally for all variables $x$ i.e to prove literally all the propositions $\mathfrak{A}(x), \mathfrak{H}(y), \mathfrak{A}(z), \ldots$ However, if we can prove $\mathfrak{A}(x)$ for a variable $x$ of a certain kind (i.e. for such $x$ which does not occur in $\mathfrak{2}(t)$ nor in any assumption of the statement $(x) \mathfrak{H}(x))$, we can see that $\mathfrak{A}(x), \mathfrak{H}(y), \mathfrak{H}(z) \ldots$ are all provable.

It is quite uncertain that $\mathscr{H}(x)$ is provable for any arbitrary variable $x$, even when $\mathfrak{H}(0), \mathfrak{H}(1), \mathfrak{H}(2), \ldots$ are surely provable, as it has been pointed out by Gödel (See Gödel [4]. See also Nishimura [8].). In formal theories, however, it is very simple to introduce universal quantification of the form $(x) \mathfrak{A}(x)$ in such a manner that the provability of $(x) \mathfrak{Y}(x)$ does not mean provability of $\mathfrak{A}(t)$ for all objects $t$ but does mean provability of $\mathfrak{A}(t)$ for all variables $t$. In fact, the majority of logical systems make their way along this line.

In our formal system too, we have to use universal quantification of the form $(x) \mathfrak{R}(x)$ in this sense. So, we call $\Gamma \mid=(x) \mathfrak{R}(x)$ provable if and only if $\Gamma \mid=\mathfrak{A}(z)$ is provable for every variable $z$.

\section{§. Practical way of description}

The leading idea of our practical way of describing formal deductions introduced in our former work (Ono [11]) is unchanged. However, our way of description exhibited here is slightly changed so as to enable easy comparison with the popular Gentzen system. (See Gentzęn [2].)

In any proof, we attach to every statemant a finite sequence of letters called the index-word ${ }^{3)}$ of the statement. We use small Greek letters as variables for finite sequences of letters (including empty case) and small German letters as variables for letters. Letters should be supplemented if necessary, and expressions such as $\alpha \beta, \alpha \ddagger \alpha\rfloor \beta$, etc. should be suitably interpreted. (More in detail, see Ono [11].)

3) The term "index-word" is originally introduced by the term "number" in Ono [11]. 
We can indicate any statement in a proof by its index-word in such manner as "the statement $(\alpha)$ ", or "the statement $(\alpha ; \mathfrak{A})$ " if we wish to denote further that $(\alpha)$ is the statement $\mathfrak{H}$. Usually, we arrange all the statements of a proof in the lexicographic order of their index-words. Accordingly, we can duely call a statement $(\alpha)$ standing before another statement $(\beta)$ in a proof if $\alpha$ stands before $\beta$ in the lexicographic order. For a fixed $\alpha$, we call the set of all the statements of the form $(\alpha \varphi)$ in a proof (including empty $\varphi$ ) the $\alpha$ block (notation: $(\alpha)^{*}$, or $(\alpha ; \mathfrak{H})^{*}$ if we wish to denote further that $(\alpha)$ is $\mathfrak{T}$ ). If $(\alpha)^{*}$ is not the unit set $\{(\alpha)\}$, we call $(\alpha)$ a lemma.

By $t_{0}, t_{1}, t_{2}, \ldots$, we denote the part of alphabetical series of letters beginning with $t$. For example, $p_{0}, p_{1}, p_{2}, \ldots$ denote $p, q, r, \ldots$ Similar notation are employed also for index-words. Namely, $\alpha_{0}, \alpha_{1}, \alpha_{2}, \ldots$ derote the index-words $\gamma t_{0}, \gamma t_{1}, \gamma t_{2}, \ldots$ assuming that $\gamma$ is $\alpha$. By $\alpha<\beta$, we denote that $\beta$ can be expressed in the forms $\alpha_{k} \delta(k \geq 1)$. Evidently, $(\alpha)$ stands before $(\beta)$ in the proof if $\alpha<\beta$.

Just as in our real inferences, every statement should be a statement either of assertion character or of assumption character. Typical examples of statements of assumption character are such statements as "Assume $\mathfrak{l}$ " or "Take any $x$ ". The former example is called a temporary assumption and the latter a temporary denomination. ${ }^{4}$

We put the index-word of a statement in a proof just before the statement. The following devices seem very practical.

(1) When $(\alpha)$ is a statement of assumption character, the last letter of $\alpha$ is written in the capital. "The statement is written in such a way as " $\alpha)(\alpha)$ ".

(2) When $(\alpha)$ is a statement of assertion character, we use only small letters for denoting $\alpha$, and the statement is written in such way as

4) The term "temporary denomination" is originally introduced by the term "temporary nomination" in Ono [11]. More popular usage of temporary denomination is such kind of denominations as "Take any $x$ satisfying $\mathfrak{A}(x)$ ". This usage can be regarded as a composition of the temporary denomination "Take any $x$ " and the temporary assumption "Assume $\mathfrak{A}(x)$ ". Because the purpose of our former work Ono [11] was practical description, we have adopted in [11] this popular usage as standard. Notice that we have also another kind of popular usage of temporary denomination of assertion character such as "Because there is at least one $t$ satisfying $\mathfrak{A}(t)$, take any one of such objects and denote it by $x$ ". We discuss denomination of this kind in $\xi 4$.

5) We use also symbols of the form $p_{n}$ for capital $\mathfrak{p}$. For example, $P_{0}, P_{1}, P_{2}, \ldots$ denote $P, Q, R, \ldots$, and $\alpha_{0}, \alpha_{1}, \alpha_{2}, \ldots$ denote $\gamma P, \gamma Q, \gamma R, \ldots$ assuming that $\alpha$ is $\gamma P$. 
a) $\quad(\alpha) / * * *$. . (if $(\alpha)$ is not a lemma.)

$\alpha)$ ) $(\alpha) / * * *$. (if $(\alpha)$ is a lemma.)

In the place "***", we give the index-words of the logical basis of this statement. (In practice, we may give the names or the numbers of already proved theorems which are used in deducing $(\alpha)$.)

It is not indispensable to give logical basis for statements of assertion character, nor to give special marks for index-words of lemmas. However, by our experience, these additional devices seem adequate for enabling easier understanding.

For any statement $(\alpha)$ in a proof, we call the set of all the statements of the form $(\alpha p)$ the frame-work of $(\alpha)^{*}$. Evidently, $(\alpha)^{*}$ is formed by $(\alpha)$ and the blocks of members of its frame-work. Lemmas only have non-empty frameworks. The set of all the statements $(\beta)$ of assumption character (except denominations) satisfying $\beta<\alpha$ is called the assumption of $(\alpha)$ as well as of $(\alpha)^{*}$.

Any logical system should have a rule for letting statements of assertion character as well as of assumption character come into proofs. The rule should be so established as to enable us to decide for any statement $(\alpha)$ of a proof only by checking the assumption and the frame-work of $(\alpha)^{*}$ whether $(\alpha)$ could come rightly into the proof.

\section{§. A primitive system of logic}

Here we introduce a primitive system of positive logic. The positive logic is supposed to be a system simplest possible under predicate logics. We describe the system along two lines, i.e. along the Gentzen line (Gentzen [2]) and along our line (Ono [11]), exhibited in $\S 2$. In this paragraph, we prove also equivalence of the systems described along these two lines.

The positive logic has two primitive notions, implication " $\supset$ " and universal quantification "( )". As for the meaning of these primitive notions, we expect that $\mathfrak{A} \supset \mathfrak{B}$ would be provable if and only if " $\mathfrak{A}$ implies $\mathfrak{B}$ " is provable and that $(x) \mathfrak{H}(x)$ would provable if and only if $\mathfrak{A}(x)$ is provable for every variable $x$.

(3.1) The system LP, a formulation of the positive logic along the Gentzen line. 
Implication " $\mid=$ ", a notion of logical level, is used for expressing sequents. Any sequent is called provable if and only if the sequent can be proved to be provable by the rules $(3.1 .1)-(3.1 .4)$ :

(3.1.1) Tautology. If a statement $\mathfrak{A}$ is a member of a statement-set $I$, then $\Gamma \mid=\mathfrak{A}$ is provable.

(3.1.2) Cut. If $\Gamma \mid=\mathfrak{A}$ and $A \cup\{\mathfrak{A}\} \mid=\mathfrak{B}$ are both provable, then $\Gamma \cup \Delta \mid=\mathfrak{B}$ is also provable.

(3.1.3) Implication rule. $\Gamma \mid=\mathfrak{A} \supset \mathfrak{B}$ is provable if and only if $\Gamma \cup\{\mathfrak{A}\} \mid=\mathfrak{B}$ is provable.

(3.1.4) Quantification rule. $\Gamma \mid=(x) \mathfrak{A}(x)$ is provable if and only if $\Gamma \mid=\mathfrak{U}(t)$ is provable for every variable $t$.

We can prove easily the following theorem.

(3.1.5) THEOREM. $\Gamma \mid=\mathfrak{A}(t)$ is provable for every variable $t$ if $\Gamma \mid=\mathfrak{A}(z)$ is provable for a variable $z$ which does not occur in $\Gamma$ and $\mathfrak{A}(t)$.

Taking this theorem into account, the inference rules (3.1.2)-(3.1.4) can be formulated as follow:

(Cut) $\frac{\Gamma|=\mathfrak{R} \quad \Lambda \cup\{\mathfrak{A}\}|=\mathfrak{B}}{\Gamma \cup \Lambda \mid=\mathfrak{B}}$.

(1 I) $\quad \frac{\Gamma \cup\{\mathfrak{A}\} \mid=\mathfrak{B}}{\Gamma \mid=\mathfrak{A} \supset \mathfrak{B}}$.

(2 I) $\frac{\Gamma \mid=\mathfrak{A} \supset \mathfrak{B}}{\Gamma \cup\{\mathfrak{M}\} \mid=\mathfrak{B}}$.

(1Q) $\frac{\Gamma \mid=\mathfrak{A}(z)}{\Gamma \mid=(x) \mathfrak{A}(x)} \quad(z$ should not occur in $\Gamma$ nor in $\mathfrak{A}(x)$.)

(2Q) $\quad \frac{\Gamma \mid=(x) \mathfrak{A}(x)}{I^{\prime} \mid=\mathfrak{A}(y)}$.

(3.1.6) We now take up another inference rule

$$
\frac{\Gamma|=\mathfrak{A} \quad \Gamma|=\mathfrak{Q} \supset \mathfrak{B}}{\Gamma !=\mathfrak{B}} .
$$

THEOREM. The single inference rule (3 I) is equivalent to the pair of inference rules (Cut) and (2 I). More precisely, any sequent is provable by tautology, (Cut), (1 I), (2 I), (1Q), and (2Q) if and only if the sequent is provable by 
tautology, (1 I), (3 I), (1 Q), and (2 Q).

We regard (1 $\mathrm{I}),(3 \mathrm{I}),(1 \mathrm{Q})$, and $(2 \mathrm{Q})$ as a system of inference rules of LP.

(3.1.7) Remark. Conjunction "." and equivalence " $\equiv$ " can be introduced in LP, though we rarely assume them in the following. Namely,

i) $\quad \frac{\Gamma|=\mathfrak{A} \quad \Gamma|=\mathfrak{B}}{\Gamma \mid=\mathfrak{A} \cdot \mathfrak{B}} . \quad \frac{\Gamma \mid=\mathfrak{A} \cdot \mathfrak{B}}{\Gamma \mid=\mathfrak{A}}, \quad \frac{\Gamma \mid=\mathfrak{A} \cdot \mathfrak{B}}{\Gamma \mid=\mathfrak{B}}$.

ii) $\quad \mathfrak{U} \equiv \mathfrak{B}$ stands for $(\mathfrak{A} \supset \mathfrak{B}) \cdot(\mathfrak{B} \supset \mathfrak{A})$.

(3.2) The system PLP, the positive logic described along our line.

The denominating quantifier ( $p$ !) means "take any variable $p$ ".

If, in a proof satisfying the following conditions $(3.2 .1)-(3.2 .3),(\alpha ; \mathfrak{l l})$ is a statement of assertion character and $\Gamma$ is the assumption of $(\alpha)^{*}$, the sequent $\Gamma \mid=\mathfrak{A}$ is called provable in PLP.

(3.2.1) Any statement of assumption character should be either a temporary assumption of the form $(\alpha \beta ; \mathfrak{H})$ or a temporary denumination of the form $(\alpha \beta ;(p !))$. In the latter case, the variable $p$ should not occur in $(\alpha)$ nor in any member of the assumption of $(\alpha \beta)^{*}$.

(3.2.2) Any statement ( $\alpha$ ) of assertion character should come in proof under one of the following categories:

\begin{tabular}{|c|c|c|c|}
\hline $\begin{array}{l}\text { A. } \\
\text { Category } \\
\text { number }\end{array}$ & $\begin{array}{l}\text { B. } \\
\text { Form of } \\
(\alpha)\end{array}$ & $\begin{array}{l}\text { C. Forms of statements which } \\
\text { should be in the union of the } \\
\text { assumption and the frame-work } \\
\text { of }(\alpha)^{*}\end{array}$ & $\begin{array}{l}\text { D. Necessary or admissible } \\
\text { statements of assumption } \\
\text { character in the frame-work } \\
\text { of }(\alpha)^{*} \text {. }\end{array}$ \\
\hline $\begin{array}{l}\text { I } \\
\text { II } \\
\text { III } \\
\text { IV }\end{array}$ & $\begin{array}{c}\mathfrak{U} \\
\mathfrak{A}(\boldsymbol{z}) \\
\mathfrak{A}=\mathfrak{B} \\
(\boldsymbol{x}) \mathfrak{A}(x)\end{array}$ & $\begin{array}{l}\mathfrak{B} \text { and } \mathfrak{B}=\mathfrak{Y} \\
(x) \mathfrak{R}(x) \\
\mathfrak{B} \\
\mathfrak{P}(z)\end{array}$ & $\begin{array}{c}\text { None } \\
\text { None } \\
\mathfrak{A}(\text { admissible }) \\
(z !)(\text { necessary. })\end{array}$ \\
\hline
\end{tabular}

(3.2.3) Thus far, for introduction of the system PLP. Now, we give some remarks concerning practical usage of PLP.

General denomination. ${ }^{6}$ To be practical, we might as well introduce the abbreviated description such as $\left(z_{0}, \cdots z_{n} !\right) \mathfrak{A}\left(z_{0}, \ldots, z_{n}\right)$ as a combination of temporary denominations and a temporary assumption. $\left(z_{0} \cdots z_{n} !\right) \mathfrak{A}\left(z_{0}, \ldots, z_{n}\right)$ is read "Take any set of variables $z_{0}, \ldots, z_{n}$ satisfying $\mathfrak{A}\left(z_{0}, \ldots, z_{n}\right)$ ".

6) See Ono [11], general nomination. See also $\S 4$ of the present paper. 
In practice, statements of the form $\left(x_{0} \cdots x_{n}\right)(5)^{7)}$ can be mostly expressed in the form $\left(x_{0} \cdots x_{n}\right)\left(\mathfrak{A}\left(x_{0}, \ldots, x_{n}\right) \supset \mathfrak{B}\left(x_{0}, \ldots, x_{n}\right)\right)$. To prove $\left(x_{0} \cdots x_{n}\right)$ in such cases, it is a usual procedure to take arbitrary variables $z_{0}, \ldots, z_{n}$ at first, and assume $\mathfrak{H}\left(z_{0}, \ldots, z_{n}\right)$ thereafter. $\left(z_{0} \cdots z_{n} !\right) \mathfrak{H}\left(z_{0}, \ldots, z_{n}\right)$ is an abbreviated description of this typical procedure. Namely, the standard description

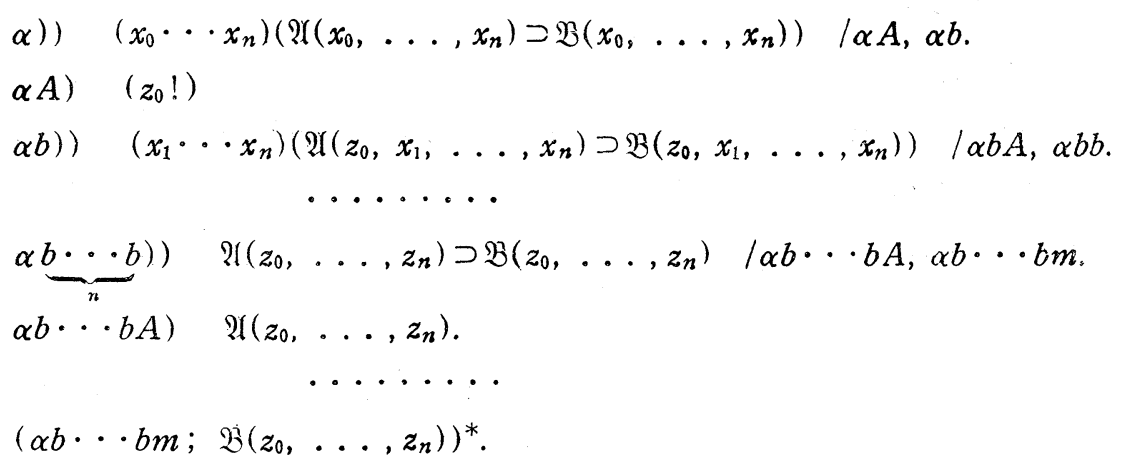

is abbreviated as

$$
\begin{gathered}
\alpha)) \quad\left(x_{0} \cdots x_{n}\right)\left(\mathfrak{A}\left(x_{0}, \ldots, x_{n}\right) \supset \mathfrak{B}\left(x_{0}, \ldots, x_{n}\right)\right) \quad / \alpha A, \alpha m . \\
\alpha A) \quad\left(z_{0} \cdots z_{n} !\right) \quad \mathfrak{A}\left(z_{0}, \ldots, z_{n}\right) . \\
\ldots \ldots \ldots \\
\left(\alpha m ; \quad B B\left(z_{0}, \ldots, z_{n}\right)\right)^{*}
\end{gathered}
$$

where every statement of the form $\left(\alpha b_{i} \varphi\right)$ is the statement $(\alpha \underbrace{b \cdots b}_{n} b_{i} \varphi)$ of the original description.

(3.2.4) Rules for conjunction, equivalence, and general denomination. Any statement of the form $\left(z_{0} \cdots z_{n} !\right) \mathfrak{U}\left(z_{0}, \ldots, z_{n}\right)$ is a statement of assumption character. If $(\alpha \beta)$ is a statement of this kind, the variables $z_{0}, \ldots, z_{n}$ should be mutually different and should not occur in $(\alpha)$ nor in any assumptions of $(\alpha \beta)^{*}$. $\quad(\alpha \beta)$ can be regarded as a temporary assumption $\mathfrak{2}\left(z_{0}, \ldots, z_{n}\right)$ for any statement $(\gamma)$ satisfying $\alpha \beta<\gamma$.

The table in (3.2.2) could be supplemented as follows:

7) $\left(x_{0} \cdots x_{n}\right) @$ stands for $\left(x_{0}\right) \cdots\left(x_{n}\right) \Subset$, 


\begin{tabular}{|c|c|c|c|}
\hline A & B & C & D \\
\hline $\begin{array}{l}\text { CI } \\
\text { CII } \\
\text { DI } \\
\text { DII }\end{array}$ & 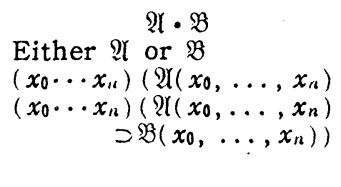 & $\begin{array}{c}\mathfrak{A} \text { and } \mathfrak{B} \\
\mathfrak{A} \cdot \mathfrak{B} \\
\mathfrak{A}\left(z_{0}, \ldots, z_{n}\right) \\
\mathfrak{B}\left(z_{0}, \ldots, z_{n}\right)\end{array}$ & $\begin{array}{c}\text { None } \\
\text { None } \\
\left(z_{0} \cdots z_{n} !\right)(\text { necessary }) \\
\left(z_{0} \cdots z_{n} !\right) \mathfrak{Q}\left(z_{0}, \ldots, z_{n}\right) \\
\text { is necessary and is regarded } \\
\text { as assumption } \mathfrak{P}\left(z_{0}, \ldots, z_{n}\right)\end{array}$ \\
\hline
\end{tabular}

(As for headings, see (3.2.2).)

$\mathfrak{A} \equiv \mathfrak{B}$ stands for $(\mathfrak{A} \supset \mathfrak{B}) \cdot(\mathfrak{B} \supset \mathfrak{U})$.

(3.3) Equivalence.

We show now equivalence of the two systems LP and PLP by proving the following two theorems.

(3.3.1) THEOREM. Any proof of a sequent in LP can be transformed into a proof of the same sequent in PLP.

Proof. Any proof of a sequent of the form $\left\{\mathfrak{A}_{1}, \ldots, \mathfrak{H}_{n}\right\} \mid=\mathfrak{B}$ in PLP is called standard, if it is a proof of the form

$$
\begin{aligned}
& \text { A) } \mathfrak{H}_{1} . \quad \text { We express this shortly by } \\
& \left.\ldots \ldots\} \quad A-A_{n-1}\right)\left\{\mathfrak{U}_{1}, \ldots, \mathfrak{A}_{n}\right\} \text {. } \\
& \left.\left.A_{n-1}\right) \mathfrak{A}_{n} .\right\} \quad(n>0) \\
& \text {.... }
\end{aligned}
$$

Now, our theorem can be proved recursively by proving the followings:

i) Every tautological sequent can be proved by a standard proof.

ii) If the assumption sequent or sequents of any inference of the types (1I), $(3 \mathrm{I}),(1 \mathrm{Q})$, and $(2 \mathrm{Q})$ are provable by standard proofs, then the conclusion sequent of the same inference can be proved by a standard proof.

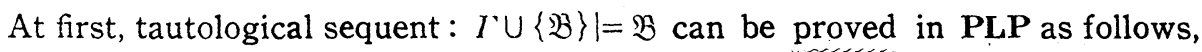
assuming $\Gamma$ contains $n$ statements.

$$
\begin{array}{llrrrr}
\left.A-A_{n-1}\right) & \Gamma . & \left.A_{n}\right) & \mathfrak{B} & \\
\left.\left.a_{n+1}\right)\right) & \mathfrak{B} \supset \mathfrak{B} & \left(a_{n+1} A .\right. & \left.a_{n+1} A\right) & \mathfrak{B} . \\
\left.a_{n+2}\right) & \mathfrak{B} & \left(A_{n},\right. & a_{n+1}
\end{array}
$$

Next, (1 I): Let $\left.\left.A-A_{n-1}\right) \Gamma . \quad A_{n}\right)$ R. $\cdots\left(a_{n+m} ; \mathfrak{B}\right)^{*}$ be a standard proof of $I \cup\{\mathfrak{T}\} \mid=\mathfrak{B}$ in PLP. Then, $\Gamma \mid=\mathfrak{Q} \supset \mathfrak{B}$ can be proved as follows : 


$$
\left.\left.\left.\left.A-A_{n-1}\right) \quad \Gamma . \quad a_{n}\right)\right) \quad \mathfrak{H} \supset \mathfrak{B} \quad / a_{n} A, a_{n} a_{m} . \quad a_{n} A\right) \quad \mathfrak{A} . \ldots \quad\left(a_{n} a_{m} ; \mathfrak{B}\right)^{*} .
$$

(Index-words $a_{n+k} \gamma(0 \leq k \leq m)$ of the former proof are transformed into $a_{n} a_{k} \gamma$ of the latter.)

Thirdly, (3 I): Let $\left.A-A_{n-1}\right) \Gamma . \quad \ldots \quad\left(a_{n+p} ; \mathfrak{B}\right)^{*} . \quad(0 \leq p) \quad$ and $\left.A-A_{n-1}\right) \Gamma \ldots \quad\left(a_{n+q} ; \mathscr{b} \supset(\mathfrak{S})^{*} .(0 \leq q)\right.$ be proofs of $\Gamma \mid=\mathfrak{B}$ and $\Gamma \mid=\mathfrak{B} \supset \mathfrak{C}$ in PLP, respectively. Then, $\Gamma \mid=\Subset$ can be proved in PLP as follows :

$$
\begin{array}{llll}
\left.A-A_{n-1}\right) & I \ldots & \ldots & \left(a_{n+p} ; \mathfrak{B}\right)^{*} \ldots \\
\left.a_{n+p+q+1}\right) & (5) & / a_{n+p}, a_{n+p+q} .
\end{array}
$$

(Index-words of the form $\boldsymbol{a}_{n+k} \gamma(0 \leq k \leq q)$ of the proof of $\Gamma \mid=\mathfrak{B} \supset(5$ are transformed into the index-words $a_{n+p+k} \gamma$ of the proof of $\Gamma \mid=(5$.)

Fourthly, (1 Q): Let $\left.A-A_{n-1}\right) \Gamma \ldots\left(a_{n+p} ;(x) \mathfrak{A}(x)\right)^{*}$ be a proof of $\Gamma \mid=(x) \mathfrak{H}(x)$ in PLP. Then, $\Gamma \mid=\mathfrak{H}(t)$ can be proved in PLP as follows :

$$
\left.\left.A-A_{n-1}\right) \Gamma \ldots \quad\left(a_{n+p} ;(x) \mathfrak{H}(x)\right)^{*} . \quad a_{n+p+1}\right) \mathfrak{A}(t) \quad / a_{n+p} .
$$

Lastly, (2Q): Let $\left.A-A_{n-1}\right) \Gamma \ldots .\left(a_{n+p} ; \mathfrak{r}(t)\right)^{*}$ be a proof of $\Gamma \mid=\mathfrak{\Re}(t)$ in PLP, having no occurrence of $t$ in $\Gamma$ as well as in $\mathfrak{f}(x)$. Then, $\Gamma \mid=(x) \mathfrak{R}(x)$ can be proved in PLP as follows:

$$
\begin{aligned}
& \left.A-A_{n-1}\right) \quad \Gamma . \\
& \left.\left.\left.a_{n}\right)\right) \quad(x) \mathfrak{R}(x) \quad / a_{n} A, a_{n} a_{p+1} . \quad a_{n} A\right) \quad(t !) . \ldots \quad\left(a_{n} a_{p+1} ; \mathfrak{\Re}(t)\right)^{*} .
\end{aligned}
$$

(Index-words of the form $a_{n+k} r(0<k<p)$ in the former proof are transformed into index-words $a_{n} a_{k+1} \gamma$ in the latter.)

(3.3.2) THEOREM. Any proof of a sequent in PLP can be transformed into a proof of the same sequent in LP.

Proof. We can prove this recursively by proving that, for any proof of a sequent $\Gamma \mid=(\alpha)$ in PLP, we can give a proof of the same sequent in LP, assuming that any proof of a sequent in PLP formed by less number of statements can be transformed into a proof of the sequent in LP.

At first, we define the reduced segment of the proof for any statement $(\varphi)$ of assertion character. Namely, if $\varphi$ is a single letter index-word $p$, then the foremost segment of the proof ending with the block $(p)^{*}$ is the reduced segment of the proof for $(p)$. If $\varphi$ is a index-word of the form $\lambda a_{h}$, then we delete the 
statement $(\lambda)$ and replace all the index-words of the form $\lambda a^{h} \theta$ by $\lambda_{h} \theta$ in the foremost segment ending with the block $(\varphi ; \mathfrak{H})^{*}$ i.e. $\left(\lambda_{h} ; \mathfrak{A}\right)^{*}$. We continue the same process again and again until the index-word of the statement $\mathfrak{F}$ becomes a single letter index-word $\mathfrak{p}$. Then, we obtain a proof in PLP ending with the block $(p ; \mathfrak{l})$ which is called the reduced segment of the proof for $(\varphi ; \mathfrak{A})$. Any reduced segment of a proof has less or equal number of statements than the original proof.

Now we transform the proof of $\Gamma \mid=(\alpha)$ in PLP into a proof of the same sequent in LP as follows, according as the category under which $(\alpha)$ comes into the proof in PLP. (As for the category numbers, see (3.2.2).)

Category I. We can find out two statements of the forms $(\beta ; \mathfrak{B} \supset \mathfrak{P})$ and $(\gamma ; \mathfrak{B})$ in the union of the assumption and the frame-work of $(\alpha)^{*}$. If $(\beta)$ (or $(\gamma)$ ) is a temporary assumption, then it belongs to $\Gamma$, so $\Gamma \mid=(\beta)$ (or $\Gamma \mid=(\gamma)$ ) is provable in LP. If $(\beta)$ (or $(\gamma)$ ) is a statement of assertion character, the reduced segement of the proof for $(\beta)$ (or for $(\gamma)$ ) is a proof in PLP formed by less number of statements than the original proof. So, we can prove in LP a segment of the form $\Gamma_{1} \mid=(\beta)$ (or of the form $\Gamma_{2} \mid=(\gamma)$ ), where $\Gamma_{1}$ (or $\Gamma_{2}$ ) is a subset of $\Gamma$. Hence, $\Gamma \mid=(\beta)$ and $\Gamma \mid=(\gamma)$ are both provable in LP anyway. Therefore, $I \mid=\mathfrak{R}$ is provable in LP by $(3 \mathrm{I})$.

Category II. $(\alpha)$ is a statement of the form $\mathfrak{B}(z)$ and we can find out a statement of the form $(\beta ;(x) B(x))$ in the union of the assumption and the frame-work of $(\alpha)$. Either $(\beta)$ is a temporary assumption, or $(\beta)$ is a statement of assertion character and the reduced segment of the proof for $(\beta)$ has less number of statements than the original proof. Hence $\Gamma \mid=(\beta)$ is provable in LP anyway. Therefore, $\Gamma \mid=(\beta)$ is provable in by $(2 \mathrm{Q})$.

Category III. $(\alpha)$ is a statement of the form $\mathfrak{N} \supset(\beta)$. Either $(\beta)$ is a tem. porary assumption, or $(\beta)$ is a statement of assertion character and the reduced segment of the proof for $(\beta)$ has less number of statements than the original proof. Hence, $I \cup\{\mathfrak{H}\} \mid=(\beta)$ is provable in LP anyway. Therefore, $I \mid=\mathfrak{N} \supset(\beta)$ is provable in LP by ( $1 \mathrm{I})$.

Category IV. $(\alpha)$ is a statement of the form $(x) \mathfrak{A}(x)$. We can find out two statements of the forms $(\beta ;(z !))$ and $(\gamma ; \mathfrak{A}(z))$ in the frame-work of $(\alpha)^{*}$ and in the union of the assumption and the frame-work of $(\alpha)^{*}$, respective- 
ly. Either $(\gamma)$ is a temporary assumption (in this case $z$ does not occur in $\mathfrak{A}(z)$ ), or $(\gamma)$ is a statement of assertion character and the reduced segment of the proof for $(\gamma)$ has less number of statements than the original proof. Hence, $I^{\prime} \mid=\mathfrak{A}(z)$ is provable in LP. Therefore, $\Gamma \mid=(x) \mathfrak{I}(x)$ is provable by $(1 \mathrm{Q})$, because $z$ does not occur in the sequent.

(3.3.3) Remark. Cut in PLP. If $\Gamma \mid=\mathfrak{A}$ and $\Lambda \cup\{\mathfrak{H}\} \mid=\mathfrak{B}$ are both provable in PLP, then $\Gamma \cup A \mid=\mathfrak{B}$ is provable in PLP.

\section{§4. Logical developments}

In this paragraph, we develop our logical systems starting from the primitive system of positive logic and assuming a system of taboos so as to be able to introduce the traditional system of logic. The set of taboos is expressed as

$$
\mathfrak{T}=\left\{\cdots, O_{i}\left(\xi_{i}\right), \cdots\right\},
$$

where $\xi_{i}$ is assumed to be a sequence of all the free variables (without repetition) in the taboo $O_{i}\left(\xi_{i}\right)$. For any $\eta_{i}$ of the same length as $\xi_{i}$, including such $\eta_{i}$ having some repetition of variables, $O_{i}\left(\eta_{i}\right)$ is regarded as a taboo. It would be adequate to suppose that some free variables may occur in a taboo as para meters (See $\$ 5$. Example.).

As shown in the preceeding paragraph, LP and PLP can be regarded as two formulations of the same system of the positive logic. Mostly, we describe our development along our line of practical description. The classical predicate logic of the first order described along this line is denoted by PLK. (See Ono [10] and [11].) However, one can easily see that the same development could be done by starting from LP and it would lead to Gentzen's LK. (See Gentzen [2]. ) This would show that PLK is equivalent to LK.

In the classical logic as well as in the intuitionistic logic, any contradiction implies any proposition. Is this valid also for taboos? Should every taboo be assumed to imply everything? It would be a matter of taste to assume this or not to do so. If we assume this, we obtain a special logical system denoted by $\mathfrak{T}$.PLJ which is closely connected with Gentzen's LJ i.e. the intuitionistic logic. (See Gentzen [2].) 疋-PLJ is formally defined in (4.2), although we do not go into details on the subject.

To introduce the logical system PLK, we do not need to go so far as to 
assume that every taboo implies everything. It is sufficient for the purpose to assume only that any taboo leads to any other taboos. The system which assumes this only is denoted by $\mathfrak{I} \cdot \mathbf{P L M}$ as it looks closely connected with the minimal logic. (See Johannson [6].) $\mathfrak{T}$-PLM is formally introduced in (4.1).

(4.1) The systems $\mathfrak{T}$-PLM and the system PLM.

(4.1.1) DEFINITION. Any sequent $\Gamma \mid=\mathfrak{H}$ is called provable in $\mathfrak{T}-\mathrm{PLM}$ if and only if a sequent of the form $\Gamma \cup \Delta \mid=\mathfrak{A}$ is provable in PLP for some set $\Delta$ formed by exclusively propositions of the form

$$
\left(\xi_{i} \xi_{j}\right)\left(O_{i}\left(\xi_{i}\right) \supset O_{j}\left(\xi_{j}\right)\right),{ }^{8)}
$$

where $\xi_{i}$ and $\xi_{i}$ are assumed to be disjoint.

We assume that our taboo system is non-void. We can fix a specially designated taboo with a fixed set of parameters, say $O .^{9)} \quad$ Then, $\Gamma \mid=\mathfrak{Q}$ is provable in $\mathfrak{T} . P L M$ if and only if a sequent of the form $\Gamma \cup A \mid=\mathfrak{A}$ is provable in PLP for a set formed by exclusively propositions of the forms $\left(\xi_{i}\right)\left(O_{i}\left(\xi_{i}\right) \supset O\right)$ or $\left(\xi_{i}\right)\left(O \supset O_{i}\left(\xi_{i}\right)\right)$.

\section{(4.1.2) Definitrion. $\rightarrow \mathfrak{A}$ stands for $\mathfrak{A} \supset O$ in $\mathfrak{I}-\mathrm{PLM}$.}

Evidently, the definition of negation can be taken as independent of the choice of the designated taboo. For, the negation $>_{1} \mathfrak{A}$ of $\mathfrak{A}$ with respect to $O_{1}$ is equivalent to the negation $>_{2} \mathfrak{A}$ of $\mathfrak{A}$ with respect to $O_{2}$ in $\mathfrak{I}$-PLM.

The following sequents are easily provable in $\mathfrak{T}-\mathbf{P L M}$.

$\begin{array}{llll}\text { (M 1) } & \varnothing \mid=>O, & (\mathrm{M} 2) & O_{i}\left(\xi_{i}\right) \mid=>\mathfrak{A}, \\ \text { (M 3) } & \{\mathfrak{H},>\mathfrak{A}\} \mid=O_{i}\left(\xi_{i}\right), & (\mathrm{M} \mathrm{4}) & \{\mathfrak{U}\} \mid=>>\mathfrak{A}, \\ \text { (M 5) } & \{>>>\mathfrak{A}\} \mid=>\mathfrak{A}, & (\mathrm{M} 6) & \{\mathfrak{A},>\mathfrak{U}\} \mid=>\mathfrak{B} .\end{array}$

(4.1.3) Any taboo system $\overline{\mathfrak{I}}$ could be called stronger than or equivalent to another taboo system $\mathfrak{I}$, if propositions provable in $\mathfrak{T}$-PLM are all provable in $\mathfrak{I}$-PLM. Accordingly, we can define “ $\mathfrak{I}$ is stronger than or equivalent to $\mathfrak{I}$ " by that sequents of the form $\emptyset \mid=\left(\xi_{i} \xi_{j}\right)\left(O_{i}\left(\xi_{i}\right) \supset O_{j}\left(\xi_{j}\right)\right)$ are provable in $\widetilde{I}$-PLM for

8) $\left(\xi_{i} \xi_{j}\right)$ stands for the multiple quantification $\left(x_{i 1} \cdots x_{i m} x_{j 1} \cdots x_{j n}\right)$ assuming that $\xi_{i}$ and $\xi_{j}$ are sequents $x_{i 1}, \ldots, x_{i m}$ and $x_{j 1}, \ldots, x_{j n}$ respectively. We assume naturally also that all $x_{\mu q}$ 's are mutually different.

9) The designated taboo can be taken also as a notation which has not already occurred in the formulation. In this case, $O$ is regarded as a new taboo added to $\mathfrak{T}$. 
any taboos $O_{i}\left(\xi_{i}\right)$ and $O_{j}\left(\xi_{j}\right)$ of $\mathfrak{T}$. If $\mathfrak{T}$ is also stronger than or equivalent to $\overline{\mathfrak{T}}$, we call them equivalent, otherwise we call $\overline{\mathfrak{I}}$ stronger than $\mathfrak{I}$.

Just as any axiom system is stronger than or equivalent to its sub-systems, any taboo system is stronger than or equivalent to its sub-taboo-systems. However, there is some difference between taboo-systems and axiom-systems in the following respect.

Starting from any axiom system $\Sigma$ we can prove theorems of $\Sigma$. If we add some theorems to the axiom system $\Sigma$ as new axioms, we get an axiom system equivalent to $\Sigma$. Similarly, referring to any taboo system $\mathfrak{I}$, we could have some contradictions which lead to some taboos. If we add these contradictions to the taboo system as new taboos, we can not always expect to have a taboo system equivalent to $\mathfrak{I}$. (Compare $(4.2 .3)$.)

(4.1.4) The system $\mathfrak{T}$-PLM can not be regarded as a purely logical system because it possibly concerns formal-theoretical statements through taboos. If the taboo system is a unit set $\{O\}$ and the only taboo $O$ is just a symbol for the designated taboo, then we have a purely logical system denoted by $O$-PLM. $O \cdot P L M$ is the weakest system under the systems IT-PLM. Of course, O-PLM is stronger than PLP.

Any system $\mathfrak{I}$-PLM is trivial if a taboo of $\mathfrak{I}$ is provable in it. The requirement for a taboo system $\mathfrak{I}$ that any taboo of $\mathfrak{I}$ should not be provable in the system I-PLM corresponds to the usual requirement for an axiom system that the axiom system should be consistent. Accordingly, we call any taboo system $\mathfrak{I}$ consistent if any taboo of $\mathfrak{I}$ is not provable in $\mathfrak{T}$-PLM. If the taboo system $\mathfrak{I}$ has only one taboo which is not provable in PLP, then the taboo system is consistent.

(4.1.5) The system PLM. A logical system called PLM is defined by the following :

(i) Logical notions of PLM are implication $\supset$, negation $\neg$, and universal quantification ( ).

(ii) In PLM, temporary assumptions and temporary denominations should be stated according to the rule for temporary assumptions and temporary denominations of PLP. (See (3.2.1).)

(iii) In PLM, statement $(\alpha)$ of assertion character should come into proof 
under any one of the following categories:

\begin{tabular}{|c|c|c|c|}
\hline $\begin{array}{l}\text { A. } \\
\text { Category } \\
\text { number }\end{array}$ & $\begin{array}{l}\text { B. } \\
\text { Form } \\
\text { of }(\alpha)\end{array}$ & $\begin{array}{l}\text { C. Forms of statements which } \\
\text { should be in the union of the } \\
\text { assumption and the frame-work } \\
\text { of }(\alpha)^{*}\end{array}$ & $\begin{array}{l}\text { D. Necessary or admissible } \\
\text { statements of assumption } \\
\text { character in the frame-work of } \\
(\alpha)^{*}\end{array}$ \\
\hline $\begin{array}{l}\text { I } \\
\text { II } \\
\text { III } \\
\text { IV } \\
\text { V }\end{array}$ & $\begin{array}{c}\mathfrak{A} \\
\mathfrak{A}(z) \\
\mathfrak{A} \supset \mathfrak{B} \\
(x) \mathfrak{A}(x) \\
\rightarrow \mathfrak{A}\end{array}$ & $\begin{array}{c}\mathfrak{B} \text { and } \mathfrak{B} \supset \mathfrak{A} \\
(x) \mathfrak{A}(x) \\
\mathfrak{B} \\
\mathfrak{A}(z) \\
\mathfrak{B} \text { and } \rightarrow \mathfrak{B}\end{array}$ & $\begin{array}{c}\text { None } \\
\text { None } \\
\mathfrak{A}(\text { admissible }) \\
(z !)(\text { necessary }) \\
\mathfrak{A}(\text { admissible })\end{array}$ \\
\hline
\end{tabular}

(Compare (3.2.2). It can be easily seen that PLM is stronger than PLP.)

(4.1.6) Theorem. PLM and O-PLM are mutually equivalent in the sense that any sequent expressible in terms of logical notions implication, negation, and universal quantification only (if we use negation symbol in O-PLM, the sequent should be expressed without the special symbol $O$ ) is provable in one system if and only if it is provable in the other.

Proof. At first, any sequent provable in PLM is also provable in O-PLM. According to (4.1.5), we have only to check the case of the category $\mathrm{V}$ after replacing every statement of the form $\neg \mathfrak{F}$ by $\mathfrak{F} \supset O$. If the statement $(\alpha)$ comes into proof under the category $\mathrm{V},(\alpha)$ is a statement of the form $(\alpha ;>\mathfrak{Y})$, and we can find out in the union of the assumption and the framework of $(\alpha)^{*}$ two statements of the forms $(\beta ; \mathfrak{B})$ and $(\gamma ;>\mathfrak{B})$. The number of members of the frame-work of $(\alpha)^{*}$ be $n$, then we can supplement the original block $(\alpha)^{*}$ so as to be a right block of a proof in O-PLM as follows:

Original block (in PLM)

$\alpha))>\mathfrak{A} / \beta, \gamma,[\alpha p]^{10)}$ $(\alpha \mathfrak{a})^{*}, \ldots,\left(\alpha \mathfrak{a}_{n-1}\right)^{*}$.
Supplemented block (in O-PLM)

$$
\begin{aligned}
& \alpha)) \quad \mathfrak{A} \supset O \quad / \alpha \mathfrak{U}_{n},[\alpha \mathfrak{p}] \\
& (\alpha \mathfrak{a})^{*}, \ldots,\left(\alpha a_{n-1}\right)^{*} \\
& \left.\alpha a_{n}\right) \quad O \quad / \beta, \gamma .
\end{aligned}
$$

Next, conversely, any sequent $I \mid=\mathfrak{A}$ provable in $O$-PLM is also provable in PLM, assuming that the sequent can be expressed in a form without $O$. To show this, take any proof of a sequent of such kind in O-PLM. Take also any fixed symbol $D$ for a proposition. Now, replace all the negative propositions

10) The notations of the form $[\varphi]$ denote the both possibilities of occurrence and nonoccurrence of the index-word $\varphi$. 
of the form $\neg \mathfrak{F}$ in the proof by $\mathfrak{F} \supset O$, and replace again all $O$ 's in the replaced proof by $>(D \supset D)$.

Every block of the proof is transformed into a right proof block in the system PLM. Hence, the sequent $I^{\prime} \mid=\mathfrak{A}$ is evidently provable in PLM if we can prove

$$
\{\neg \mathfrak{F}\} \mid=\mathfrak{F} \supset \neg(D \supset D) \text { and }\{\mathfrak{F} \supset \neg(D \supset D)\} \mid=>\mathfrak{F} \text {, }
$$

which are both provable in PLM without difficulty.

(4.1.7) Remark. In PLM too, if two sequents $\Gamma \mid=\mathfrak{A}$ and $\Lambda \cup\{\mathfrak{A}\} \mid=\mathcal{B}$ are both provable, then the sequent $\Gamma \cup \Lambda \mid=\mathfrak{B}$ is also provable (Cut rule in PLM).

Further, we would like to remark here that the sequents

$$
\begin{aligned}
& \{\neg \neg(\neg \mathfrak{A} \supset>\mathfrak{B})\} \mid=\neg \mathfrak{A} \supset>\mathfrak{B}, \\
& \{>\neg(x)>\mathfrak{A}(x)\} \mid=(x)>\mathfrak{A}(x),
\end{aligned}
$$

and

$$
\{\mathfrak{H} \supset \neg \mathfrak{B}\} \mid=>\neg \mathfrak{A} \supset \neg \mathfrak{B}
$$

are all provable in PLM.

(4.1.8) Remark. We can introduce $\forall, \exists, \rightarrow, \wedge, \vee$ of $\mathfrak{I}-$ PLK or PLK in $\mathfrak{I}$-PLM or PLM. (Compare (4.3.7).). Namely, $\forall x \cdot \mathfrak{H}(x)$ by $(x)>\neg \mathfrak{H}(x)$ (proved to be epuivalent to $\neg \neg(x)>\neg \mathfrak{A}(x)$ by $(4.1 .7)), \exists x \cdot \mathfrak{H}(x)$ by $\neg(x)>\mathfrak{A}(x), \quad \mathfrak{A} \rightarrow \mathfrak{B}$ by $\neg \neg \mathfrak{A} \supset \neg \neg \mathfrak{B} \quad$ (proved to be equivalent to $\neg \neg(\neg \neg \mathfrak{A} \supset \neg \neg \mathfrak{B})$ by (4.1.7)), $\mathfrak{A} \wedge \mathfrak{B}$ by $\neg(\mathfrak{A} \supset \neg \mathfrak{B})$ (proved to be equvalent to $\neg(\neg \neg \mathfrak{A} \supset \neg \mathfrak{B})$ by $(4.1 .7))$, and $\mathfrak{A} \vee \mathfrak{B}$ by $\neg \mathfrak{H} \supset \neg \neg \mathfrak{B}$ (proved to be equivalent to $\neg \neg(\neg \mathfrak{A} \supset \neg \neg \mathfrak{B})$ by (4.1.7)).

(4.2) The systems $\mathfrak{I}-\mathbf{P L J}$ and the system PLJ.

(4.2.1) DeFinition. Any sequent $\Gamma \mid=\mathfrak{A}$ is called provable in $\mathfrak{T}-\mathbf{P L J}$, if and only if a sequent of the form $\Gamma \cup \Delta \mid=\mathfrak{A}$ is provable in PLP for some set $\Delta$ formed exclusively by propositions of the form $\left(\xi_{i} \eta\right)\left(O_{i}\left(\xi_{i}\right) \supset \mathfrak{B}\left(\eta_{\eta}\right)\right)$, assuming that all the variables in the sequence $\xi_{i} \eta$ are mutually diffierent.

(4.2.2) We can define negation in any $\mathfrak{T}$-PLJ system just as in $\mathfrak{I}$-PLM. We can also define the system $O$-PLJ and the system PLJ just as we have defined O-PLM and PLM. O-PLJ and PLJ are mutually equivalent in the 
sense of (4.1.6). Clearly, $\mathfrak{T}-\mathbf{P L J}$ is stronger than $\mathfrak{T}-\mathbf{P L M}$ for any taboo system $\mathfrak{T}$.

(4.2.3) If $\tilde{\mathfrak{T}}$-PLJ provability implies $\mathfrak{T}-\mathbf{P L J}$ provability for every sequent, then $\mathfrak{I}$ could be called $\mathbf{J}$-stronger than or $\mathbf{J}$-equivalent to $\mathfrak{I}$. If, moreover, $\mathfrak{I}$ is $\mathbf{J}$-stronger or $\mathbf{J}$-equivalent to $\widetilde{\mathfrak{I}}$, the two taboo systems $\mathfrak{I}$ and $\widetilde{\mathfrak{I}}$ are called $\mathbf{J}$ equivalent to each other. The necessary and sufficient condition for $\widetilde{\mathfrak{I}}$ to be $\mathbf{J}$ stronger than or $\mathbf{J}$-equivalent to $\mathfrak{I}$ is that any sequent of the form $O_{i} \mid=\mathfrak{H}$ is provable in $\widetilde{\mathfrak{I}}-\mathbf{P L J}$ for any taboo $O_{i}$ of $\mathfrak{T}$.

Now, let $\mathfrak{I}$ be any taboo system and $\widehat{S}$ be a contradiction in $\mathfrak{I}$-PLJ i.e. $\{\Subset\} \mid=O_{i}$ be provable for some taboo $O_{i}$ in $\mathfrak{T}$-PLJ. If $\tilde{\mathfrak{I}}$ is a set of all the taboos of $\mathfrak{I}$ and some contradictions of this kind, then $\mathfrak{I}$ is $\mathbf{J}$-equivalent to $\mathfrak{I}$. (Compare (4.1.3).)

(4.3) The systems $\mathfrak{I}$-PLK and the system PLK.

The traditional logic is a logic of the world of possible objects. Objects are assumed to be existing because their existence can not be denied, statements are asserted because they can not be denied. In accordance with this circumstance, we try to introduce a system, in which all the statements can be regarded to have double negative character. Logical notions would have to be suitably modified to keep the inference rule of $\mathfrak{T}$-PLM. In fact, such a plan can be realized, and the imagined system is called $\mathfrak{T}$.PLK. We express sequents of $\mathfrak{I}$-PLK by the form $\Gamma \mid-\mathfrak{U}$ which is interpreted as $\bar{\Gamma} \mid=>\neg \mathfrak{U}$ in $\mathfrak{I}$-PLM, where $\bar{\Gamma}$ is the set of statements $\neg \neg \sqrt{ }$ for all members $\Subset$ of $\Gamma$. Implication and universal quantification can be suitably modified so as to keep all the inference rules of $\mathfrak{I}$-PLM valid. Modified implication is denoted by the symbol $\rightarrow$ and modified universal quantification is denoted by the symbol $\forall$. Conjunction, disjunction, equivalence, and existential quantification can be defined in this system by implication and universal quantification of PLP along the usual line. Most remarkable feature of the system is that $\{\neg \neg \mathfrak{U}\} \mid-\mathfrak{A}$ is always provable.

Corresponding to $O$-PLM and PLM, we can define equivalent (in the sense of (4.1.6)) systems, $O$-PLK and PLK. To be practical, we really give the inference rule of PLK later.

(4.3.1) Sequents of $\mathfrak{T}-$ PLK and PLK are expressed in the form 
$\left\{\mathfrak{A}_{1}, \ldots, \mathfrak{A}_{n}\right\} \mid-\mathfrak{B}$ which means $\left\{\neg \neg \mathfrak{A}_{1}, \ldots,>>\mathfrak{A}_{n}\right\} \mid=>\neg \mathfrak{B}$.

(4.3.2) To make $\Gamma \cup\{\mathfrak{A}\} \mid-\mathfrak{B}$ and $\Gamma \mid-\mathfrak{A} \rightarrow \mathfrak{B}$ equivalent, we define $\mathfrak{A} \rightarrow \mathfrak{B}$ by $\neg \neg \mathfrak{A} \supset \neg \neg \mathfrak{B}$.

To make $\Gamma \mid-\forall x \mathfrak{A}(x)$ equivalent to that $\Gamma \mid-\mathfrak{H}(z)$ holds for every variable $z$, we define $\forall x \cdot \mathfrak{A}(x)$ by $(x)>\neg \mathfrak{A}(x)$.

(4.3.3) Remark. Evidently, $\Gamma \mid-\mathfrak{A}$ and $\Lambda \cup\{\mathfrak{U}\} \mid-\mathfrak{B}$ lead to $\Gamma \cup A \mid-\mathfrak{B}$ in T-PLK.

(4.3.4) THEOREM. $\quad \Gamma \mid-\mathfrak{A} \rightarrow \mathfrak{B}$ is provable in $\mathfrak{T}-\mathbf{P L K}$ if and only if $\Gamma \cup\{\mathfrak{A}\} \mid-\mathfrak{B}$ is provable in $\mathfrak{T} \cdot \mathbf{P L K} . \quad \Gamma-\forall x \cdot \mathfrak{A}(x)$ is provable $\mathfrak{T}-\mathbf{P L K}$ if and only if $\Gamma \mid-\mathfrak{H}(z)$ is provable for every variable $z$.

We can prove the theorem by making use of (4.1.2)-(M 4), (4.1.4), (4.1.7), (4.3.1), and (4.3.2).

(4.3.5) THEOREM. We can not prove any taboo in $\mathfrak{I}-\mathbf{P L K}$, i.e. $\emptyset \mid-O_{i}$ is not provable in $\mathfrak{T}-\mathbf{P L K}$, as long as $\mathfrak{I}$ is consistent.

Proof. If $\varnothing \mid-O_{i}$ is provable, then $\varnothing \mid=>>O_{i}$ i.e. $\varnothing \mid=\left(O_{i} \supset O\right) \supset O$ must be provable in $\mathfrak{I}$-PLM. Hence, $\varnothing \mid=O$ must be provable in $\mathfrak{T}$-PLM.

(4.3.6) The system PLK. Theoretically, we can define PLK only by replacing $\supset$ and () of the table in (4.1.5) by $\rightarrow$ and $\forall$ respectively and by adding the following category VI to the table.

\begin{tabular}{|c|c|c|c|}
\hline $\mathrm{A}$ & $\mathrm{B}$ & $\mathrm{C}$ & $\mathrm{D}$ \\
\hline $\mathrm{VI}$ & $\mathfrak{A}$ & $\mathfrak{B}$ and $\rightarrow \mathfrak{B}$ & $\neg \mathfrak{M}$ (admissible) \\
\hline
\end{tabular}

(As for the headings, see (4.1.5).) For PLJ (intuitionistic logic), one should take instead of this category the category $\mathrm{VI}^{*}$ which is obtained from this category by substituting "None" in place of " $\rightarrow \mathfrak{U}$ (admissible)" under the heading $D$.)

Practically, however, it is far more convenient to introduce a few other logical notions and supplement the table for these notions. We shall give a table of such kind in (4.3.7).

Our present concern is to show that PLK defined here is equivalent to $O$ - 
PLK as far as statements expressible in terms of $\rightarrow$ and $\forall$ concern. We can prove this as follows:

Firstly, the following is easily proved to hold in $O$-PLK: If two sequents of the forms $\Gamma \cup\{\neg \mathfrak{A}\} \mid-\mathfrak{B}$ and $\Gamma \cup\{\neg \mathfrak{A}\} \mid-\neg \mathfrak{B}$ are both provable, then $\Gamma \mid-\mathfrak{A}$ is also provable. Hence, any sequent provable in PLK is also provable in $O$. PLK.

Next, conversely, let $\left\{\mathfrak{A}_{1}, \ldots, \mathfrak{U}_{n}\right\} \mid-\mathfrak{B}$ be a sequent provable in $O$-PLK. Then, the sequent $\left\{\neg \neg \mathfrak{A}_{1}^{*}, \ldots,>>\mathfrak{A}_{n}^{*}\right\} \mid=\neg \neg \mathfrak{B}^{*}$ is provable in O.PLM, where $\mathfrak{F}^{*}$ denotes the formula obtained from $\mathfrak{F}$ by expressing $\rightarrow$ and $\forall$ in terms of $\supset$ and ( ) according to the definition (4.3.2). Now, let us denote by $\mathfrak{F}^{* *}$ the formula obtained by replacing all the symbols $\supset$ and $($ ) occurring in $\mathfrak{F}^{*}$ by $\rightarrow$ and $\forall$, respectively. Then, we can prove easily that the sequents, $\left\{>>\mathfrak{A}_{1}^{* *}, \ldots,>>\mathfrak{A}_{n}^{* *}\right\}\left|->>\mathfrak{B}^{* *}, \quad\left\{\mathfrak{H}_{i}\right\}\right|->>\mathfrak{A}_{1}^{* *} \quad(i=1,2, \ldots, n)$, $\left\{\neg \neg \mathfrak{B}^{* *}\right\} \mid-\mathfrak{B}$ are all provable in PLK. (By (4.3.2) and (4.3.6).) This shows that the sequent $\left\{\mathfrak{A}_{1}, \ldots, \mathfrak{A}_{n}\right\} \mid-\mathfrak{B}$ is provable in PLK.

(4.3.7) Other logical notions. In any $\mathfrak{T}$-PLK system as well as in PLK, we can introduce logical notions such as disjunction $\vee$, conjunction $\wedge$, equivalence $\equiv$, and existential quantification $\exists$ by defining them as usual in terms of $\rightarrow, \rightarrow$, and $\forall$. (Compare (4.1.8).) Concerning equivalence, we should

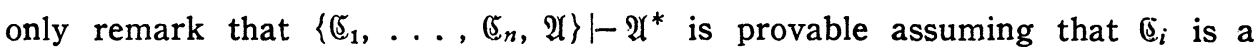
statement of the form $\forall \hat{\xi}_{i}\left(\widetilde{\mho}_{i}\left(\xi_{i}\right) \equiv \mathfrak{\mho}_{i}\left(\xi_{i}\right)\right)$ and $\mathfrak{H}^{*}$ denotes a statement obtained from $\mathfrak{A}$ by replacing some of sub-formulas of the forms $\xi_{i}\left(\xi_{i}\right)$ by $\tilde{\mho}_{i}\left(\xi_{i}\right)$.

Existential quantification can be defined as usual (i.e. $\exists x \mathfrak{Y}(x)$ stands for $\neg \forall x \cdot \neg \mathfrak{U}(x)$.) However, in PLK, we can introduce temporary denominations of the form $\exists z ! \mathfrak{A}(z)$ of assertion character, which means "There is an object $t$ satisfying $\mathfrak{A}(t)$, so take any one of such object $t$ and call it $z$." Inference rules for the existential quantification and the existential denominating quantification are given in the following table together with the rules for conjunction and disjunction. The variable $z$ which is introduced by an existential denominating quantification $(\alpha ; \exists z ! \mathfrak{H}(z))$ should not occur in $\mathfrak{A}(t)$ nor in the assumption of $(\alpha)$. Further, $(\alpha ; \exists z ! \mathfrak{F}(z))$ should be treated as the statement $\mathfrak{A}(z)$ with the free variable $z$ in references of statements standing after $(\alpha)$. 


\begin{tabular}{|c|c|c|c|}
\hline $\begin{array}{l}\text { A. } \\
\text { Category } \\
\text { number }\end{array}$ & $\begin{array}{l}\text { B. } \\
\text { Forms } \\
\text { of }(\alpha)\end{array}$ & $\begin{array}{l}\text { C. Forms of statements which } \\
\text { should be in the union of } \\
\text { assumption and the frame-work } \\
\text { of }(\alpha)\end{array}$ & $\begin{array}{l}\text { D. Necessary or admissible } \\
\text { statements of assumption } \\
\text { character in the frame-work of } \\
(\alpha)\end{array}$ \\
\hline $\begin{array}{l}\text { I } \\
\text { II } \\
\text { III } \\
\text { IV } \\
\text { V } \\
\text { VI } \\
\text { VII } \\
\text { VIII } \\
\text { IX } \\
\text { X } \\
\text { XI } \\
\text { XII }\end{array}$ & $\begin{array}{l}\mathfrak{A} \\
\mathfrak{A}(z) \\
\mathfrak{A} \rightarrow \mathfrak{B} \\
\forall x \mathfrak{A}(x) \\
\overrightarrow{\mathfrak{A}}^{\mathfrak{A}} \\
\mathfrak{A} \wedge \mathfrak{B} \\
\mathfrak{A} \\
\mathfrak{A} \vee \mathfrak{B} \\
\mathfrak{A} \\
\exists x \cdot \mathfrak{A}(x) \\
\exists z ! \mathfrak{A}(z)\end{array}$ & $\begin{array}{l}\mathfrak{B} \text { and } \mathfrak{B} \rightarrow \mathfrak{U} \\
\forall x \cdot \mathfrak{H}(x) \\
\mathfrak{B} \\
\mathfrak{H}(z) \\
\mathfrak{B} \text { and } \rightarrow \mathfrak{B} \\
\mathfrak{B} \text { and } \overrightarrow{\mathfrak{B}} \\
\mathfrak{A} \text { and } \mathfrak{B} \\
\text { Either } \mathfrak{A} \wedge \mathfrak{B} \text { or } \mathfrak{B} \wedge \mathfrak{A} \\
\text { Etiher } \mathfrak{A} \text { or } \mathfrak{B} \\
\mathfrak{B} \vee \mathfrak{C}, \mathfrak{B} \rightarrow \mathfrak{A} \text { and } \mathfrak{C} \rightarrow \mathfrak{A} \\
\mathfrak{A}(z) \\
\exists x \cdot \mathfrak{A}(x)\end{array}$ & $\begin{array}{l}\text { None } \\
\text { None } \\
\mathfrak{A} \text { (admissible) } \\
\forall z !(\text { necessary) } \\
\mathfrak{A} \text { (admissible) } \\
\rightarrow \mathfrak{A} \text { (admissible) } \\
\text { None } \\
\text { None } \\
\text { None } \\
\text { None } \\
\text { None } \\
\text { None }\end{array}$ \\
\hline
\end{tabular}

(One should take the category $\mathrm{VI}^{*}$ instead of VI for PLJ. One should delete VI for PLM (minimal logic).)

The categories VII-XII are not necessary if we define $\wedge, \vee$, and $\exists$ in terms of $\rightarrow, \neg$, and $\forall$. Further, we can prove conversely that the definitions of $\wedge, \vee$, and $\exists$ turn out dispensable if we adopt the categories VII-XII. ${ }^{11)}$

As it seems that no further illustration on the matter is necessary for the definitions of $\wedge$ and $V$, or the categories VII-X, we will prove only that the definition of $\exists$ is equivalent to the categories XI and XII.

At first, we prove that the categories XI and XII leads to equivalence of $\exists x \cdot \mathfrak{U}(x)$ and $>\forall x \cdot \neg \mathfrak{A}(x)$ :

Proof of $\exists x \cdot \mathfrak{I}(x) \mid-\neg \forall x \cdot>\mathfrak{A}(x) \quad /$ A, c.
A) $\exists x \cdot \mathfrak{U}(x)$.
b) $\exists z ! \mathfrak{U}(z) / \mathrm{A}$.
c)) $>\forall x \cdot \neg \mathfrak{A}(x) \quad / \mathrm{b}, \mathrm{cb}$.
cA) $\forall x \cdot \neg \mathfrak{O}(x)$,
$\mathrm{cb}) \quad \neg \mathfrak{A}(z) \quad / \mathrm{cA}$.

Proof of $>\forall x \cdot \rightarrow \mathfrak{A}(x) \mid-\exists x \cdot \mathfrak{U}(x) \quad / \mathrm{A}, \mathrm{b}$.

A) $>\forall x \cdot \neg \mathfrak{A}(x)$.

b)) $\exists x \cdot \mathfrak{A}(x) \quad / \mathrm{bA}, \mathrm{A}, \mathrm{bb}$.

bA) $>\exists x \cdot \mathfrak{H}(x)$

bb)) $\forall x \cdot>\mathfrak{A}(x) \quad /$ bbA, bbb.

bbA) $\forall z !$.

bbb)) $\rightarrow \mathfrak{U}(z)$ /bbbb, bA. bbbA) $\mathfrak{Y}(z)$. bbbb) $\exists x \cdot \mathfrak{A}(x) \quad /$ bbbA.

Accordingly, any sequence provable by the definition of the existential quantifier can be proved by the categories XI and XII. Next, conversely,

11) We can introduce general existential denomination of the form $\exists x \cdots z ! \mathfrak{A}(x, \ldots, z)$ in a natural way. ISee (3.2.3).). We can further introduce more general mixed denominations of the form $\mathbf{Q}_{1} x_{1} \ldots \mathbf{Q}_{n} x_{n} ! \mathfrak{M}\left(x_{1}, \ldots, x_{n}\right)$, where $\mathbf{Q}_{i}$ is either of $\forall$ or $\exists$. (See Ono [11], general nomination.). 
we will show that any sequent provable by the categories XI and XII are also provable by the definition.

If the statement $(\alpha)$ comes into a proof by the category XI, $(\alpha)$ is a statement of the form $\exists x \cdot \mathfrak{A}(x)$, and there is a statement of the form $(\beta ; \mathfrak{Y}(z))$ in the union of the assumption and the frame-work of $(\alpha ; \exists x \cdot \mathfrak{I}(x))^{*}$. We transform the block $(\alpha)^{*}$ of the proof as follows so as to become a proof depending on the definition of existential quantifier:

Original block

$$
\alpha)) \quad \exists x \cdot \mathfrak{A}(x) \quad / \beta \text {. }
$$$$
\ldots,\left(\alpha a_{n-1}\right)^{*} \text {. }
$$

$(n \geq 0 . \alpha))$ should be

replaced by $\alpha$ ) if $n=0$ )
Transformed block

a)) $\exists x \cdot \mathfrak{A}(x)$ i.e. $\neg \forall x \cdot \neg \mathfrak{U}(x) / \alpha A_{n}, \alpha a_{n+1}, \beta$. ... $\left.\alpha a_{n-1}\right)^{*}$.

$\left.\alpha A_{n}\right) \quad \forall x \cdot \neg \mathfrak{Y}(x)$

$\left.\alpha a_{n+1}\right) \quad \neg \mathfrak{U}(z) \quad / \alpha A_{n}$.

Now we discuss the case where $(\alpha)$ comes into a proof by the category XII. If $(\alpha)$ is a lemma and the frame-work of $(\alpha)^{*}$ has $n$-members, then replace the index-word $\alpha$ by $\alpha_{n}$, all the index-words of the form $\alpha a_{i} \varphi$ by $\alpha_{i} \varphi$ $(i=0, \ldots, n-1)$, and all the index-words of the form $\alpha_{k} \varphi$ by $\alpha_{k+n} \varphi(k \geq 1)$. The replaced proof is easily seen to be a right proof. For non-lemma $(\alpha)$, we can take $n$ as 0 . In the replaced proof, $\left(\alpha_{n}\right)$ is a statement of the form " $\alpha) \exists z ! \widetilde{F}(z) / \gamma$ " where $(\gamma)$ is a statement standing before $(\alpha)$. In the replaced proof, we express the index-word $\alpha$ in the form $\beta \mathrm{m}$, and we transform the block $(\beta)^{*}$ so as to be a right block of proof based on the definition of the existential quantifier by $>$ and $\forall$. If $\beta$ is a vacant sequence, $(\beta)^{*}$ denotes the whole proof and $(\beta)$ denotes the conclusion of the sequent proved by the proof.

We transform the proof separately in the following four cases.

At first, the case where $(\beta)$ comes into proof by the categories V or VI. In this case, the block $(\beta)^{*}$ of the form
ß)) $>\mathfrak{A}($ or $\mathfrak{A}) \quad / *, * *,[* * *]$.
$\beta m) \quad \exists z ! \mathfrak{F}(z) / \gamma \cdot \cdots$

is transformed into

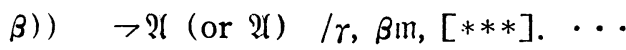

$$
\begin{aligned}
& \beta m)) \quad \forall x \cdot \neg \mathfrak{F}(x) \quad / \beta m A, \beta m b . \quad \beta m A) \quad \forall z \text { ! }
\end{aligned}
$$


$\beta \mathrm{mb})) \quad \neg \mathfrak{F}(z) \quad(\beta \mathrm{mbA}, *, * * . \quad \beta \mathrm{mbA}) \quad \mathfrak{F}(z) . \cdots$

All the statements of the form $\left(\beta \mathfrak{m}_{i} \varphi\right)$, if there are any, should be placed after $(\beta \mathrm{mb})$ by changing their index-words to $\beta \mathrm{mba}_{i-1} \varphi(i \geq 1)$.

Next, if $(\beta)$ comes into proof $\quad$ (i) by the category III,

(ii) by the category IV, (iii) by the categories other than III-VI, then $(\beta)^{*}$ is a block of the form

(i) $\beta$ ) $) \quad \mathfrak{A} \rightarrow \mathfrak{B} \quad / *,[* *] . \quad \cdots \quad \beta \mathrm{m}) \quad \exists z ! \mathfrak{F}(z) / \gamma . \cdots$

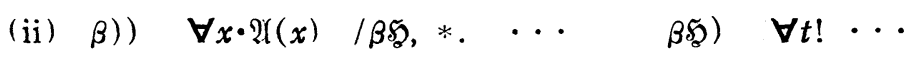
$\beta m) \quad \exists z ! \mathfrak{F}(z) / \gamma . \quad \cdots$

(iii) $\beta$ ) ) (f $/ *, \cdots, \cdots, \beta$ n) $\exists z ! \mathfrak{F}(z) / \gamma . \cdots$

respectively. We can transform these into

(i) $\beta$ ) ) $\mathfrak{N} \rightarrow \mathfrak{B} \quad /[* *], \beta m_{1}, \cdots, \quad(\beta m ;-\forall x \cdot \neg \mathfrak{F}(x) \rightarrow \mathfrak{B})^{*}$. $\left.\beta m_{1}\right) \quad \mathfrak{B} / \gamma, \beta m$.

(ii) $\beta$ ) ) $\left.\forall x \cdot \mathfrak{A}(x) \quad / \beta \mathscr{S}_{2}, \beta \mathfrak{m}_{1} . \quad \cdots \quad \beta \mathscr{S}_{2}\right) \quad \forall t$ ! $\quad \cdots$ $\left.(\beta m ; \rightarrow \forall x \cdot \rightarrow \mathfrak{F}(x) \rightarrow \mathfrak{F}(t))^{*} . \quad \beta m_{1}\right) \quad \mathfrak{H}(t) \quad / \gamma, \beta m$.

(iii) $\beta)) \quad$ If $/ \gamma, \beta m . \cdots \quad(\beta \mathrm{m} ; \rightarrow \forall x \cdot \rightarrow \mathfrak{F}(x) \rightarrow \mathfrak{A})^{*}$,

respectively. All the blocks $(\beta \mathrm{m})^{*}$ of these proofs are blocks of the form $\left(\beta m ; \rightarrow \forall x \cdot \rightarrow \mathfrak{F}(x) \rightarrow(5)^{*}\right.$, so we can show the construction of these blocks simultaneously as follows:

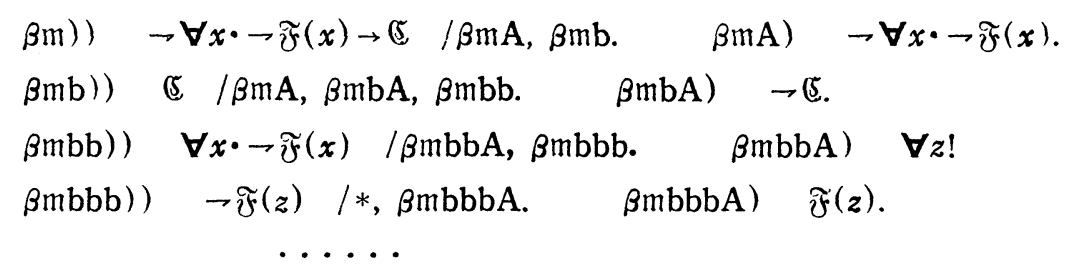

The statements after $(\beta \mathrm{mbbbA})$ in this block are the statements after $(\beta \mathrm{m})$ in the block $(\beta \mathrm{m})^{*}$ of the original proof obtained by changing every indexwords of the form $\beta m_{i} \varphi$ to the index-words $\beta \mathrm{mbbba}_{i} \varphi$. The transformed blocks remain as right blocks as far as the original blocks $(\beta)^{*}$ are right blocks.

(4.3.8) Remark. Instead of starting from $\mathfrak{T}$-PLM, we could also start from $\mathfrak{I}$-PLJ. "By doing so, we could have obtained a system $\mathfrak{T}$-PLK* which is perfectly similar to $\mathfrak{I}$-PLK. Concerning $\rightarrow, \wedge, \vee, \rightarrow, \forall, \exists$, these two systems 
do not show any difference. We have prefered $\mathfrak{T}$-PLM to $\mathfrak{T}$-PLJ for our starting system, because we have wished to start from a system having as weak assumption as possible. (See (5.2). Compare Kuroda [7].)

\section{(5) Examples}

Many formal theories are developable starting from PLP and a taboo system. In fact, as is shown in (5.1), any mathematical theory might be developable along this line, if we can find out a taboo expressible in terms of $\supset$ and ( ) only in the system. However, in the real course of our thinking, this might be stupid if the theory becomes complicated by doing so. Especially, we would like to expect a simple expression of the disignated taboo. Peano's axiom system of natural numbers looks like a nice example of a theory having a simple desig. nated taboo. (See (5.2).)

(5.1) Trivial taboo systems of theories defined by axiom system.

Let $\Sigma$ be a usual formal theory defined by an axiom-system $\left\{\cdots, \mathfrak{A}_{i}\right.$, $\cdots\}$ in the classical predicate logic of the first order with logical symbols $\wedge, \vee, \rightarrow, \forall, \exists$, and let $O$ be a special proposition symbol which does not occur in any axiom. Then, we can trivally interpret the axioms in terms of $\supset,()$, and $O$ by the rules given in (4.1.8). (Take $\rightarrow \mathscr{F}$ as standing for $\mathfrak{F} \supset O$.) Let the formula obtained from $\mathfrak{A}_{i}$ by this interpretation be denoted by $\mathfrak{A}_{i}^{*}$, then the set $\widetilde{\jmath}$ of formulas $\mathfrak{H}_{i}^{*} \supset O$ can be regarded as a taboo system of $\Sigma$.

Now, let $\tilde{I}$ be a system of taboos which can be expressed in terms of $\wedge, \vee, \rightarrow, \forall, \exists$ only, and $\mathfrak{I}$ be a system of formulas expressible in terms of $\supset$, ( ) (if one wish, the symbol introduced in (3.1.7) could be added) obtained from members of $\tilde{\mathfrak{I}}$ by replacing $\vee, \wedge, \rightarrow, \forall, \exists$ partly by the rule given in (4.1.8) and partly $\supset$ in place of $\rightarrow$ and ( ) in place of $\forall$ (if one wish, partly - in place of $\wedge$ ). Then, any proposition provable in $\mathfrak{I}-\mathbf{P L K}$ is also provable in $\widetilde{\mathfrak{I}}$-PLK (every taboo in $\widetilde{I}$ be regarded as a formula expressed in terms of $\supset$ and ( ) by the rules given in (4.1.8).). For, if all the symbols $\supset$ and ( ) in taboos of $\mathfrak{T}$ are replaced again by $\rightarrow$ and $\forall$ respectively, then we would have a new taboo system $\mathfrak{F}^{*}$ which is easily proved to be weaker than $\widetilde{\mathfrak{I}}$.

(5.2) Taboo system of natural numbers.

Peano's axiom system can be formulated in PLK as follows: (We disregard here theories of recursive functions because our purpose is only to show a 
simple example of taboo systems.)

P 1. $O \neq x^{\prime}$

P 2. $x=x$

P 3. $x=y \rightarrow(\mathfrak{A}(x) \rightarrow \mathfrak{A}(y))$,

P 4. $x^{\prime}=y^{\prime} \rightarrow x=y$,

P 5. $(x)\left(\mathfrak{H}(x) \rightarrow \mathfrak{U}\left(x^{\prime}\right)\right) \rightarrow(\mathfrak{H}(O) \rightarrow \mathfrak{A}(z))$,

where $O$, ', and $=$ are taken as primitive notions. As the taboo system of the primitive theory of this system, we can take

PT 1. $x=x \supset O=O^{\prime}$

PT 2. $\left(x^{\prime}=y^{\prime} \supset x=y\right) \supset O=O^{\prime}$

PT 3. $\left(x=y \supset(\mathfrak{A}(x) \supset \mathfrak{A}(y)) \supset O=O^{\prime}\right.$

PT 4. $\left((x)\left(\mathfrak{R}(x) \supset \mathfrak{F}\left(x^{\prime}\right)\right) \supset(\mathfrak{A}(O) \supset \mathfrak{Q}(z))\right) \supset O=z^{\prime}$.

It is easy to show that $O=O^{\prime}$ can be regarded as the designated taboo of the system. (See (4.1.3).)

It should be noticed that these axioms are simply expressed in terms of $\supset$ and ( ).

(5.3) On taboos of set theories.

In most set theories, any statement of the form $x \in x$ can be taken as a taboo. This is valid even in the theories of sets and classes, e.g. BernaysGödel set theory (Bernays [1], Gödel [5].). Regarding $x \in x$ as the only taboo scheme, $p \subseteq q$ might be as well interpreted to mean " $p$ is distinct from $q$ ".

This taboo system could be much strengthened by taking up all the formulas of the form

$$
(p)((s t)(s \in t \cdot t \in p: \supset s \in p) \cdot x \in p: \supset x \in x)
$$

as taboos, which practically implies existence of an infinite ascending series $a_{0} \in u_{1} \in a_{2} \in \cdots$ without any cycle (i.e. $i \leq j$ implies $\rightarrow a_{j} \in a_{i}$ ). Here, formulas of the form $\mathfrak{T} \cdot \mathfrak{B}: \supset \mathbb{S}$ stands for $\mathfrak{A} \supset(\mathfrak{B} \supset()$.

\section{REFERENCES}

[1] Bernays, P. A system of axiomatic set theory, Part I-VII; Symbolic Logic; I, vol. 2 (1937), pp. 65-77; II, vol. 6 (1941), pp. 1-17; III, vol. 7 (1942), pp. 65-89; IV, vol. 7 (1942), pp. 133-145; V, vol. 8 (1943), pp. 89-106; VI, vol. 13 (1948), pp.65-79; VII, vol. 
19 (1954), pp. 81-96.

[2] Gentzen, G. Untersuchungen über das logischen Schliessen, Math. Z., 39, pp. 176-210, 405-431 (1935).

[3] Glivenko, V. Sur quelques points de la logique de M. Brouwer, Academie Royale de Belgique, Bull. de la classe des sciences, ser. 5, 15 (1929), pp. 183-188.

[4] Gödel, K. Über formal unentscheidbare Sätze der Principia Mathematica und verwandter Systeme I. Monatsch. f. Math. u. Phys., vol. 38 (1931), pp. 173-198.

[5] Gödel, K. The consistency of the axiom of choice and of the generalized continuumhypothesis with the axioms of set theory, Annals of Math. Studies, No. 3, Princeton.

[6] Johansson, I. Der Minimalkalkül, ein reduzierter intutionistischer Formalismus, Compositio Mathematicae, vol. 4 (1936), pp. 119-136.

[7] Kuroda, S. Intuitionistische Untersuchungen der Formalistischen Logik, Nagoya. Math. J., vol. 2 (1951), pp. 35-47.

[ 8 ] Nishimura, T. On Gödel's theorem, J. Math. Soc. Jap., vol. 13 (1961), pp. 1-12.

[9] Ono, K. A theory of mathematical objects as a prototype of set theory, Nagoya Math. J., vol. 20 (1962), pp. 106-168.

[10] Ono, K. A stronger system of object theory as a prototype of set theory, Nagoya Math. J., vol. 22 (1963), pp. 119-167.

[11] Ono, K. On a practical way of describing formal deductions, Nagoya Math. J., vol. 21 (1962), pp. 115-121.

[12] Suetuna, Z. Über die Grundlagen der Mathematik I-III: I, J. Math. Soc. Jap., vol. 3 (1951), pp. 59-68: II, III, Proc. Jap. Academy: II, vol. 27 (1951), pp. 389-392: III, vol. 29 (1953), pp. 91-95.

\section{Mathematical Institute}

Nagoya University 